Research, Society and Development, v. 10, n. 16, e185101622836, 2021

(CC BY 4.0) | ISSN 2525-3409 | DOI: http://dx.doi.org/10.33448/rsd-v10i16.22836

\title{
Análise das intervenções utilizadas na prevenção e controle da hemorragia pós-parto: revisão integrativa da literatura
}

Analysis of the interventions used in the prevention and control of postpartum hemorrhage: an integrative literature review

Análisis de las intervenciones utilizadas en la prevención y el control de la hemorragia posparto: una revisión integradora de la literatura

Recebido: 04/11/2021 | Revisado: 20/11/2021 | Aceito: 29/11/2021 | Publicado: 11/12/2021

Melissa Torres Soares Rabêlo

ORCID: https://orcid.org/0000-0002-5344-9894 Centro Universitário de Ciências e Tecnologia do Maranhão, Brasil

E-mail: melissatdss11@gmail.com

Ana Carla Marques da Costa

ORCID: https://orcid.org/0000-0002-4246-145X Centro Universitário de Ciências e Tecnologia do Maranhão, Brasil E-mail: carlama271@gmail.com

Amanda Kauany Pereira da Silva

ORCID: https://orcid.org/0000-0003-3317-1570 Faculdade Tecnológica e Educação Superior Profissional, Brasil

E-mail: kauanyamanda003@gmail.com Joyce Soares de Araújo ORCID: https://orcid.org/0000-0002-2543-4636 Universidade de Fortaleza, Brasil E-mail: joycejoyce1996@gmail.com

Kessya Karynne de Araújo Silva ORCID: https://orcid.org/0000-0002-3797-7566 Centro Universitário de Ciências e Tecnologia do Maranhão, Brasil E-mail: karynne21.ka@gmail.com

Lara Beatriz de Sousa Coelho ORCID: https://orcid.org/0000-0002-8640-7172 Centro Universitário de Ciências e Tecnologia do Maranhão, Brasil E-mail: larabiacoelho@gmail.com

Eduardo Brito da Silva

ORCID: https://orcid.org/0000-0002-8571-7806

Centro Universitário de Ciências e Tecnologia do Maranhão, Brasil E-mail: eduzinhobds@gmail.com

Luana Pereira Ibiapina Coêlho ORCID: https://orcid.org/0000-0002-2054-959X Universidade Estadual do Maranhão, Brasil

E-mail: luana_ibiapina@ hotmail.com

Jesineide Sousa da Silva

ORCID: https://orcid.org/0000-0003-2930-1694 Centro Universitário de Ciências e Tecnologia do Maranhão, Brasil

E-mail: jesineides@gmail.com

Adriana Borges Ferreira da Silva ORCID: https://orcid.org/0000-0002-9000-1298 Centro Universitário de Ciências e Tecnologia do Maranhão, Brasil

E-mail: ferreiraalves.adri@gmail.com

Larissa Lima Marques Coimbra

Universidade Federal do Maranhão, Brasil ORCID: https://orcid.org/0000-0001-7827-9871

E-mail: coimbralarissa@gmail.com

Rosinei Nascimento Ferreira ORCID: https://orcid.org/0000-0003-2732-7778 Instituto de Gestão Educacional Signorelli, Brasil

E-mail: rosineifff2@hotmail.com

Pedro Vitor Mendes Santos

ORCID: https://orcid.org/0000-0002-2249-1440

Universidade Estadual do Maranhão, Brasil E-mail: pedrovitorp2@hotmail.com 


\author{
Eudilene da Silva Mesquita \\ ORCID: https://orcid.org/0000-0001-9894-0903 \\ Centro Universitário de Ciências e Tecnologia do Maranhão, Brasil \\ E-mail: eu.mesquitta@gmail.com \\ Jeíse Rodrigues Belarmino \\ ORCID: https://orcid.org/0000-0002-7566-7024 \\ Universidade Federal do Maranhão, Brasil \\ E-mail: jeiserodrigues120@hotmail.com
}

\begin{abstract}
Resumo
A hemorragia pós-parto (HPP) é definida como uma perda de sangue $\geq 500 \mathrm{~mL}$ que acontece nas 24 horas após o parto, quando essa perda é maior que $1.000 \mathrm{~mL}$ é considerada grave. A HPP está entre as principais causas de morbidades maternas graves, responsável por cerca de $25 \%$ de todas as mortes de gestantes no mundo, sobretudo em países de baixa renda. No Brasil ocupa a segunda maior causa de morte materna. Este estudo teve como objetivo analisar a produção científica acerca das intervenções utilizadas na prevenção e controle da hemorragia pós-parto. Tratou-se de uma pesquisa bibliográfica do tipo revisão integrativa da literatura, os estudos foram extraídos das bases de dados PubMed (National Library of Medicine); BVS (Biblioteca Virtual da Saúde) e CINAHL (Cumulative Index to Nursing and Allied Health Literature). Utilizou-se 14 estudos publicados nos anos de 2016 à 2021 . O nível de evidencia predominante foi II - ensaio clínico randomizado, 11 estudos obtiveram grau de recomendação A. Os resultados concluem que, as intervenções analisadas como efetivas para a prevenção da hemorragia pós-parto foram: ocitocina, misoprostol, carbetocina, ergometrina ou metilergometrina, o manejo ativo no terceiro período do trabalho de parto e a ligadura da artéria uterina. No controle da HPP foram: o ácido tranexâmico, tamponamento com balão uterino (BTU) e o traje anti-choque não pneumático (NASG).
\end{abstract}

Palavras-chave: Hemorragia pós-parto; Prevenção e controle; Efetividade das intervenções; Enfermagem obstétrica.

\begin{abstract}
Postpartum hemorrhage (PPH) is defined as a blood loss $\geq 500 \mathrm{~mL}$ that occurs within 24 hours after delivery, when this loss is greater than $1,000 \mathrm{~mL}$ it is considered severe. PPH is among the leading causes of severe maternal morbidities, accounting for about $25 \%$ of all deaths of pregnant women worldwide, especially in low-income countries. In Brazil, it is the second leading cause of maternal death. This study aimed to analyze the scientific production on interventions used to prevent and control postpartum hemorrhage. This was a bibliographic research of the integrative literature review type; studies were extracted from the PubMed (National Library of Medicine), BVS (Virtual Health Library) and CINAHL (Cumulative Index to Nursing and Allied Health Literature) databases. We used 14 studies published from 2016 to 2021. The predominant level of evidence was II - randomized clinical trial, 11 studies obtained grade recommendation $\mathrm{A}$. The results conclude that the interventions analyzed as effective for the prevention of postpartum hemorrhage were: oxytocin, misoprostol, carbetocin, ergometrine or methylergometrine, active management in the third period of labor and uterine artery ligation. In the control of PPH were: tranexamic acid, uterine balloon tamponade (BTU) and the non pneumatic anti-shock suit (NASG).
\end{abstract}

Keywords: Postpartum hemorrhage; Prevention and control; Effectiveness of interventions; Obstetric nursing.

\title{
Resumen
}

La hemorragia posparto (HPP) se define como una pérdida de sangre $\geq 500 \mathrm{ml}$ que ocurre dentro de las 24 horas posteriores al parto, cuando esta pérdida es superior a $1000 \mathrm{ml}$, se considera grave. La HPP se encuentra entre las principales causas de morbilidad materna grave y representa alrededor del $25 \%$ de todas las muertes de mujeres embarazadas en todo el mundo, especialmente en los países de bajos ingresos. En Brasil, se ubica como la segunda causa principal de muerte materna. Este estudio tuvo como objetivo analizar la producción científica sobre intervenciones utilizadas en la prevención y control de la hemorragia posparto. Esta fue una investigación bibliográfica de tipo revisión de literatura integradora, los estudios fueron extraídos de las bases de datos de PubMed (Biblioteca Nacional de Medicina); BVS (Biblioteca Virtual en Salud) y CINAHL (Índice acumulativo de literatura de enfermería y salud afín). Se utilizaron 14 estudios publicados en los años 2016 a 2021. El nivel de evidencia predominante fue II - ensayo clínico aleatorizado, 11 estudios obtuvieron grado de recomendación A. Los resultados concluyen que las intervenciones analizadas como efectivas para la prevención del parto post-hemorrágico fueron: oxitocina, misoprostol, carbetocina, ergometrina o metilergometrina, manejo activo en el tercer período de parto y ligadura de arteria uterina. En el control de la HPP estuvieron: ácido tranexámico, taponamiento uterino con balón (BTU) y traje antichoque no neumático (NASG).

Palabras clave: Hemorragia post parto; Prevención y control; Efectividad de las intervenciones; Enfermería obstétrica. 


\section{Introdução}

A hemorragia pós-parto (HPP) é conceituada como uma perda sanguínea superior a $500 \mathrm{ml}$, mensurada no período de 24 horas após o parto. A HPP é uma das principais causas de morbidades maternas graves no mundo, como internações hospitalares prolongadas, necessidade de transfusões sanguíneas e procedimentos cirúrgicos que levam à perda da função reprodutiva. Responsável por cerca de $25 \%$ de todas as mortes de gestantes no mundo, sobretudo em países de baixa renda. Ocorrem 14 milhões de casos de hemorragia pós-parto todos os anos. Desse grupo, 140 mil mulheres morrem por causa da hemorragia, o que representa uma morte a cada 4 minutos (Felipe et al., 2020; Finlayson et al., 2019).

No Brasil a HPP ocupa a segunda maior causa de morte materna, perdendo apenas para os distúrbios hipertensivos. Apesar da ampliação do acesso aos serviços de saúde e da melhoria em praticamente todos os indicadores de saúde materna, a hemorragia continua se apresentando entre os três principais grupos de causas de morte no ciclo grávido-puerperal, sendo a atonia uterina e a anormalidade na placentação as etiologias específicas mais frequentes (Martins \& Silva, 2018; Oliveira \& Davim, 2019).

Dados da Organização Mundial de Saúde (OMS) mostraram que, embora os índices de mortes maternas tenham caído em todo o mundo, continua sendo um problema relevante do setor da saúde. Não somente por serem mortes evitáveis, mas esses índices refletem a situação social em que vivem as mulheres, também por demonstrarem as fragilidades com a qualificação do atendimento no período gravídico-puerperal e a organização dos serviços de saúde (World Health Organization [WHO], 2018).

Rangel et al. (2019), ressaltam que, certamente todas as instituições e profissionais de saúde que manejam mulheres em trabalho de parto, terão contato em algum momento, com casos de hemorragia pós-parto. Dessa forma, os profissionais e os serviços de saúde devem conhecer e colocar em suas práticas assistenciais, as tecnologias com maior índice de evidência. Mesmo assim, o nível de evidência para prevenção e tratamento da HPP é globalmente baixo.

Este estudo teve como problemática: Quais evidências científicas sobre a efetividade das intervenções utilizadas na prevenção e controle da hemorragia pós-parto?

Assim, a relevância deste estudo, consiste na necessidade constante de buscar novas informações, necessárias e atualizadas no que se refere às intervenções utilizadas para prevenir e tratar a hemorragia pós-parto, a qual resulta em altos índices de morbimortalidade materna mundialmente. Bem como, proporcionar norteamento aos profissionais atuantes diante da problemática, contribuindo para a melhoria da assistência e possibilitando atualização a respeito do assunto através do conhecimento das práticas baseadas em evidências.

Dessa forma a pesquisa justifica-se por tratar-se de uma temática pouco divulgada e discutida, com quantidade insuficiente de publicações científicas voltadas para a efetividade das intervenções em questão. Portanto, determinar as estratégias preventivas e de controle mais eficazes é crucial. Pois a intervenção adequada, utilizada em tempo ágil, pode ter um forte impacto na redução significativa da mortalidade materna. Para tal, esse estudo teve como objetivo analisar a produção científica acerca das intervenções utilizadas na prevenção e controle da hemorragia pós-parto.

\section{Metodologia}

Este estudo trata-se de uma pesquisa bibliográfica do tipo revisão integrativa da literatura. Este procedimento foi escolhido por possibilitar a síntese e análise do conhecimento científico já produzido sobre o tema "Análise das intervenções utilizadas na prevenção e controle da hemorragia pós-parto". Esta revisão utilizou a metodologia proposta no estudo de

Oliveira et al. (2016). 
De acordo com Ercole et al. (2014), a revisão integrativa de literatura é um método que tem como finalidade sintetizar resultados obtidos em pesquisas de maneira sistemática, ordenada e abrangente, mediante diferentes metodologias. É denominada integrativa porque fornece informações mais amplas sobre um assunto, constituindo um corpo de conhecimento e podendo ser direcionada para a definição de conceitos, revisão de teorias ou análise metodológica dos estudos. Este método proporciona a combinação de dados da literatura teórica e empírica, proporcionando maior compreensão do tema de interesse.

O tema, determinou a construção da estratégia PICO, que representa um acrônimo para Paciente (P), Intervenção (I), Comparação (C) e Desfechos (O-outcomes), na qual foi utilizada para a geração da questão norteadora desta revisão integrativa da literatura: "Quais evidências científicas sobre a efetividade das intervenções utilizadas na prevenção e controle da hemorragia pós-parto?"

Para a localização dos estudos que respondessem à pergunta de pesquisa, utilizou-se de descritores indexados e não indexados (palavras-chave) nos idiomas português, inglês e espanhol. Os descritores foram obtidos a partir do Medical Subject Headings (MESH), dos Descritores em Ciências da Saúde (DeCS) e dos títulos CINAHL, como mostra o Quadro 1.

Quadro 1. Elementos da estratégia PICO, descritores e palavras-chave utilizados. Caxias, MA, Brasil, 2021.

\begin{tabular}{|c|c|c|c|c|}
\hline \multicolumn{2}{|r|}{ ELEMENTOS } & MESH & DECS & TÍTULOS CINAHL \\
\hline $\mathbf{P}$ & "Hemorragia Pós-Parto" & "Postpartum Hemorrhage" & "Postpartum Hemorrhage" & "Postpartum Hemorrhage" \\
\hline I & $\begin{array}{l}\text { "Efetividade das } \\
\text { Intervenções" }\end{array}$ & $\begin{array}{c}\text { "Clinical Trial" } \\
\text { OR } \\
\text { "Comparative Effectiveness } \\
\text { Research" }\end{array}$ & $\begin{array}{c}\text { "Clinical Trial" } \\
\text { OR } \\
\text { "Methods" } \\
\text { OR } \\
\text { "Disease Management" }\end{array}$ & $\begin{array}{c}\text { Management OR Treatment OR } \\
\text { Intervention OR Therapy }\end{array}$ \\
\hline $\mathbf{C}$ & - & - & - & - \\
\hline $\mathbf{O}$ & $\begin{array}{l}\text { "Prevenção e Controle da } \\
\text { Hemorragia Pós-Parto" }\end{array}$ & $\begin{array}{c}\text { "Prevention and Control" } \\
\text { OR } \\
\text { "Postpartum Hemorrhage" }\end{array}$ & $\begin{array}{c}\text { "Prevention \& control" } \\
\text { OR } \\
\text { "Postpartum Hemorrhage" }\end{array}$ & $\begin{array}{c}\text { "Postpartum Prevention and } \\
\text { Control" }\end{array}$ \\
\hline
\end{tabular}

Fonte: Descritores Mesh e Decs e Títulos CINAHL (2021).

O elemento C da estratégia PICO não foi abordado nesta pesquisa pois esta não tem por objetivo comparar intervenções. Os termos utilizados durante a pesquisa foram classificados e combinados nos bancos de dados, resultando em estratégias específicas de cada base.

Consultou-se por meio de descritores e palavras-chave as bases de dados PubMed da National Library of Medicine, Cumulative Index to Nursing and Allied Health Literature (CINAHL) e Biblioteca Virtual da Saúde (BVS), coordenada pela BIREME e composta de bases de dados bibliográficas produzidas pela Rede BVS, como Literatura científica e técnica da América Latina e Caribe (LILACS), Scientific Electronic Library Online (SciELO), manuais da Organização Pan-Americana da Saúde (OPAS), Organização Mundial da Saúde (OMS) e outras fontes de informação (Quadro 2).

Quadro 2. Estratégias de busca utilizadas nas bases de dados BIREME, PUBMED e CINAHL. Caxias, MA, Brasil, 2021.

\begin{tabular}{|c|c|c|c|c|}
\hline Base de dados & Estratégia de busca & Resultados & Filtrados & Selecionados \\
\hline $\begin{array}{c}\text { BIREME } \\
\text { (Descritores Decs) }\end{array}$ & $\begin{array}{l}\text { (Postpartum Hemorrhage) AND (Clinical Trial OR methods OR } \\
\begin{array}{l}\text { Disease Management) AND (prevention \& control OR } \\
\text { postpartum hemorrhage) }\end{array}\end{array}$ & 463 & 128 & 6 \\
\hline $\begin{array}{c}\text { PUBMED } \\
\text { (descriptors MeSH) }\end{array}$ & $\begin{array}{l}\text { (postpartum hemorrhage) AND (clinical trial OR comparative } \\
\text { effectiveness research) AND (prevention and control OR } \\
\text { postpartum hemorrhage) }\end{array}$ & 519 & 62 & 5 \\
\hline $\begin{array}{l}\text { CINAHL } \\
\text { (CINAHL } \\
\text { Headings) }\end{array}$ & $\begin{array}{l}\text { (hemorrhage postpartum) AND (management or treatment or } \\
\text { intervention or therapy) AND (Postpartum Prevention and } \\
\text { Control) }\end{array}$ & 603 & 108 & 3 \\
\hline
\end{tabular}

Fonte: Bases de dados (2021). 
Como critérios de inclusão, foram selecionados estudos disponíveis sobre a temática em sua totalidade, publicados nos últimos cinco anos, de 2016 a 2021, nos idiomas Português, Espanhol e Inglês. Foram excluídos da busca inicial, resumos, textos incompletos, teses, dissertações, relatos técnicos, estudos de caso e outras formas de publicação que não fossem artigos científicos completos.

A análise para seleção dos estudos foi realizada em duas fases, a saber:

Na primeira, os estudos foram pré-selecionados segundo os critérios de inclusão e exclusão e de acordo com a estratégia de funcionamento e busca de cada base de dados.

Foram encontrados quatrocentos e sessenta e três (463) estudos como busca geral na BVS, sendo que limitando a busca para artigos com texto completo realizado com humanos nos últimos cinco anos, obteve-se cento e vinte e oito (128) estudos, destes foram analisados títulos e resumos onde apenas seis (6) estudos foram condizentes com a questão desta pesquisa.

Na base de dados PUBMED, como busca total foram encontrados quinhentos e dezenove (519) estudos, aplicando na pesquisa o filtro que limita por texto completo dos últimos cinco anos com humanos, obteve-se sessenta e dois (62) estudos, destes foram analisados títulos e resumos e teve como resultado final cinco (5) estudos.

$\mathrm{Na}$ CINAHL foram obtidos seiscentos e três (603) estudos como busca geral, sendo que limitando a busca para artigos com texto completo realizado nos últimos cinco anos com humanos, obteve-se cento e oito (108) estudos, sendo três (3) foram condizentes com a questão desta pesquisa após a análise dos títulos e resumos.

$\mathrm{Na}$ segunda fase os estudos foram analisados quanto ao potencial de participação no estudo, avaliando o atendimento à questão de pesquisa, bem como o tipo de investigação, objetivos, amostra, método, desfechos, resultados e conclusão, resultando em quatorze (14) artigos.

$\mathrm{Na}$ análise e interpretação dos resultados, foram coletadas informações dos artigos científicos e criadas categorias analíticas que facilitaram a ordenação e a sumarização de cada estudo. Essa categorização foi realizada de forma descritiva, indicando os dados mais relevantes para o estudo.

A pesquisa levou em consideração os aspectos éticos da pesquisa quanto às citações dos estudos, respeitando a autoria das ideias, os conceitos e as definições presentes nos artigos incluídos na revisão. As evidências científicas foram classificadas segundo os níveis e graus de recomendação propostos por Bork (2005).

\section{Resultados}

\subsection{Descrição dos estudos}

Dos quatorze estudos incluídos nesta revisão, (13/92,9\%) estavam na língua inglesa, $(1 / 7,1 \%)$ na língua portuguesa. A maioria das publicações foram concentradas no ano de 2019 (06/42,8\%) com abordagem quantitativa (14/100\%); com relação aos periódicos a Cochrane database of systematic reviews (03/21,4\%) foi o que mais forneceu estudos para esta amostra; o nível de evidência predominante foi II (ensaio clínico randomizado), também foram inclusas revisões sistemáticas, estudo de coorte e meta-análise; 11 estudos (78,6\%) obtiveram grau de recomendação “A” para mudança na prática clínica; Os EUA/Reino Unido/Egito foram os países com mais estudos incluídos (6/42,9\%), conforme demonstrado na Tabela 1. 
Tabela 1. Análise descritiva das produções científicas acerca das intervenções utilizadas na prevenção e controle da hemorragia pós-parto. Caxias, MA, Brasil, $2021(\mathrm{~N}=14)$.

\begin{tabular}{|c|c|c|}
\hline Variáveis & $\mathbf{N}$ & $\%$ \\
\hline \multicolumn{3}{|l|}{ Base de dados } \\
\hline BIREME & 6 & 42,9 \\
\hline PUBMED & 5 & 35,7 \\
\hline CINAHL & 3 & 21,4 \\
\hline \multicolumn{3}{|l|}{ Abordagem do estudo } \\
\hline Qualitativo & - & - \\
\hline Quantitativo & 14 & 100,0 \\
\hline \multicolumn{3}{|l|}{ Delineamento da pesquisa } \\
\hline Revisão Sistemática & 04 & 28,7 \\
\hline Ensaio clínico randomizado & 08 & 57,1 \\
\hline Estudo de Coorte & 01 & 7,1 \\
\hline Meta-análise & 01 & 7,1 \\
\hline \multicolumn{3}{|l|}{ Idioma } \\
\hline Inglês & 13 & 92,9 \\
\hline Português & 01 & 7,1 \\
\hline \multicolumn{3}{|l|}{ Nível de evidência } \\
\hline Um & 02 & 14,3 \\
\hline Dois & 12 & 85,7 \\
\hline \multicolumn{3}{|l|}{ Grau de Recomendação } \\
\hline A & 11 & 78,6 \\
\hline B & 03 & 21,4 \\
\hline \multicolumn{3}{|l|}{ Distribuição temporal } \\
\hline 2016 & 02 & 14,3 \\
\hline 2017 & 02 & 14,3 \\
\hline 2018 & 02 & 14,3 \\
\hline 2019 & 06 & 42,8 \\
\hline 2020 & 02 & 14,3 \\
\hline \multicolumn{3}{|l|}{ Periódicos } \\
\hline Cochrane Database Syst Ver & 03 & 21,4 \\
\hline BJOG: An International Journal of Obstetrics and Gynaecology & 02 & 14,3 \\
\hline Revista Lat Am Enfermagem & 01 & 7,1 \\
\hline Obstetrics and gynecology & 01 & 7,1 \\
\hline Journal of Maternal-Fetal \& Neonatal Medicine & 01 & 7,1 \\
\hline The Lancet & 01 & 7,1 \\
\hline BMC Pregnancy \& Childbirth & 01 & 7,1 \\
\hline Basic \& Clinical Pharmacology \& Toxicology & 01 & 7,1 \\
\hline Archives of Gynecology and Obstetrics & 01 & 7,1 \\
\hline International Journal of Gynecology \& Obstetrics & 01 & 7,1 \\
\hline NIHR Journals Library & 01 & 7,1 \\
\hline \multicolumn{3}{|l|}{ Procedência } \\
\hline Brasil & 01 & 7,1 \\
\hline Colômbia & 01 & 7,1 \\
\hline EUA & 02 & 14,3 \\
\hline Egito & 02 & 14,3 \\
\hline Irlanda & 01 & 7,1 \\
\hline França & 01 & 7,1 \\
\hline Reino Unido & 02 & 14,3 \\
\hline Turquia & 01 & 7,1 \\
\hline Tailândia & 01 & 7,1 \\
\hline Suíça & 01 & 7,1 \\
\hline Paquistão & 01 & 7,1 \\
\hline
\end{tabular}

Legenda: N= número; \%= percentual. Fonte: Artigos pesquisados (2021).

Os estudos avaliaram a aplicação de intervenções na prevenção e tratamento da hemorragia pós-parto (HPP). Foram apresentadas as principais intervenções analisadas nos estudos para o manejo da HPP, comparando sua efetividade com outras intervenções e/ou placebo. Os estudos analisaram a eficácia do uso de medicamentos uterotônicos, manejo ativo do terceiro estágio do trabalho de parto, tamponamento uterino com balão, traje anti-choque não pneumático, ligadura da artéria uterina na prevenção e/ou controle da HPP, como mostra o Quadro 3 abaixo. 


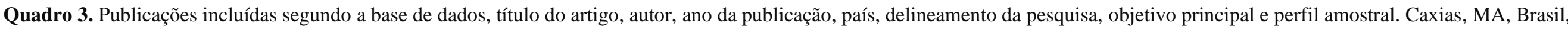

$2021(\mathrm{~N}=14)$.

\begin{tabular}{|c|c|c|c|c|}
\hline $\begin{array}{l}N^{0} \text { de } \\
\text { ordem }\end{array}$ & Título do artigo & Autor/ano & Objetivo principal & Perfil amostral \\
\hline $\begin{array}{c}\text { A1 } \\
\text { BIREME }\end{array}$ & $\begin{array}{l}\text { Tecnologias de cuidado para prevenção e } \\
\text { controle da hemorragia no terceiro estágio do } \\
\text { parto: revisão sistemática. }\end{array}$ & Rangel et al., 2019. & $\begin{array}{l}\text { Identificar evidências acerca das contribuições das } \\
\text { tecnologias de cuidado usadas para prevenção e } \\
\text { controle da hemorragia no terceiro estágio do parto. }\end{array}$ & $\begin{array}{l}\text { Busca em bases de dados com a adoção do fluxograma PRISMA } \\
\text { (Preferred Reporting Items for Systematic Reviews and Meta-Analyses), } \\
\text { encontraram-se } 6.999 \text { artigos e foram incluídos como corpo de análise } 42 \\
\text { artigos. Destes, } 33 \text { analisaram a eficácia das drogas uterotônicas para } \\
\text { prevenção/ tratamento da HPP. }\end{array}$ \\
\hline $\begin{array}{c}\text { A2 } \\
\text { BIREME }\end{array}$ & $\begin{array}{l}\text { Prophylactic oxytocin for the third stage of } \\
\text { labour to prevent postpartum haemorrhage. }\end{array}$ & $\begin{array}{l}\text { Salati, Leathersich, } \\
\text { Williams, Cuthbert e } \\
\text { Tolosa, } 2019 .\end{array}$ & $\begin{array}{l}\text { Determinar a eficácia da ocitocina profilática para } \\
\text { prevenir HPP e outros resultados maternos adversos na } \\
\text { terceira fase do trabalho de parto. }\end{array}$ & $\begin{array}{l}\text { Revisão inclui } 24 \text { estudos, com } 23 \text { estudos envolvendo } 10.018 \text { mulheres } \\
\text { contribuindo com dados. Devido a muitos ensaios avaliadas em alto risco } \\
\text { de viés, grau de evidência variou de muito baixa a moderada qualidade. }\end{array}$ \\
\hline $\begin{array}{c}\text { A3 } \\
\text { BIREME }\end{array}$ & $\begin{array}{l}\text { Active versus expectant management for } \\
\text { women in the third stage of labour. }\end{array}$ & Begley et al. (2019) & $\begin{array}{l}\text { Comparar os efeitos do manejo ativo versus expectante } \\
\text { do terceiro estágio do trabalho de parto na hemorragia } \\
\text { pós-parto primária grave (HPP) e outros resultados } \\
\text { maternos e infantis. }\end{array}$ & $\begin{array}{l}\text { Foram incluídos oito estudos, envolvendo análise de dados de } 8.892 \\
\text { mulheres. Todos os estudos foram realizados em hospitais, sete em países } \\
\text { de alta renda e um em um país de baixa renda. }\end{array}$ \\
\hline $\begin{array}{c}\text { A4 } \\
\text { BIREME }\end{array}$ & $\begin{array}{l}\text { Effectiveness of Per Rectal Misoprostol } \\
\text { Versus Intramuscular Oxytocin for Prevention } \\
\text { of Primary Postpartum Haemorrhage. / }\end{array}$ & Asmat et al. (2017) & $\begin{array}{l}\text { Comparar a eficácia do misoprostol retal em relação à } \\
\text { ocitocina na hemorragia pós-parto primária (HPP). }\end{array}$ & $\begin{array}{l}\text { O tamanho da amostra calculado foi } 1678 \text {, ( } 839 \text { para cada grupo), } \\
\text { mantendo a proporção da população prevista para tomar misoprostol como } \\
40,5 \% \text { e populaça prevista proporção para tomar oxitocina como } 50 \% \text {, } \\
\text { nível de confiança } 95 \% \text { e poder do teste } 90 \% \text {. }\end{array}$ \\
\hline $\begin{array}{c}\text { A5 } \\
\text { BIREME }\end{array}$ & $\begin{array}{l}\text { Active Management of the Third Stage of } \\
\text { Labor With a Combination of Oxytocin and } \\
\text { Misoprostol to Prevent Postpartum } \\
\text { Hemorrhage: A Randomized Controlled Trial. }\end{array}$ & Quibel et al. (2016) & $\begin{array}{l}\text { Avaliar a eficácia e segurança do misoprostol } \\
\text { administrado simultaneamente com a ocitocina como } \\
\text { parte do manejo ativo do terceiro estágio do parto. }\end{array}$ & $\begin{array}{l}\text { Este estudo multicêntrico, duplo-cego, randomizado e controlado por } \\
\text { placebo recrutou mulheres no primeiro estágio do trabalho de parto com } \\
\text { partos vaginais esperados em 36-42 semanas de gestação, incluindo } 1.721 \\
\text { pacientes. }\end{array}$ \\
\hline $\begin{array}{c}\text { A6 } \\
\text { BIREME }\end{array}$ & $\begin{array}{l}\text { Uterine balloon tamponade for the treatment } \\
\text { of postpartum hemorrhage: a systematic } \\
\text { review and meta-analysis. }\end{array}$ & Suarez et al. (2020) & $\begin{array}{l}\text { Avaliar a eficácia, efetividade e segurança do } \\
\text { tamponamento com balão uterino para o tratamento de } \\
\text { hemorragia pós-parto. }\end{array}$ & $\begin{array}{l}\text { Bancos de dados eletrônicos (desde o início até agosto de 2019) e } \\
\text { bibliografias. Foram incluídos } 91 \text { estudos, incluindo } 4729 \text { mulheres, } \\
\text { preencheram os critérios de inclusão ( } 6 \text { estudos randomizados, } 1 \text { estudo } \\
\text { randomizado de cluster, } 15 \text { estudos não randomizados e } 69 \text { séries de } \\
\text { casos). }\end{array}$ \\
\hline $\begin{array}{l}\text { PUBMED } \\
07\end{array}$ & $\begin{array}{l}\text { Impact of nonpneumatic antishock garment in } \\
\text { the management of patients with } \\
\text { hypoperfusion due to massive postpartum } \\
\text { hemorrhage. / }\end{array}$ & Escobar et al. (2020) & $\begin{array}{l}\text { Comparar os parâmetros hemodinâmicos e de perfusão, } \\
\text { bem como os resultados clínicos em pacientes } \\
\text { criticamente enfermos com HPP que receberam } \\
\text { tratamento com um traje anti-choque não pneumático } \\
\text { (NASG) como parte de um pacote de intervenção, com } \\
\text { um grupo de pacientes em condições semelhantes que } \\
\text { não receberam NASG. }\end{array}$ & $\begin{array}{l}\text { O estudo analisou uma coorte histórica de } 154 \text { pacientes com HPP, } \\
\text { choque hipovolêmico secundário e sinais de hipoperfusão internados nesta } \\
\text { instituição de } 2012 \text { a } 2015 \text {. Grupo } 1(n=77) \text { tratado com NASG e Grupo } \\
2(n=77) \text { recebeu outras intervenções além do NASG. }\end{array}$ \\
\hline $\begin{array}{l}\text { PUBMED } \\
08\end{array}$ & $\begin{array}{l}\text { The Effect of the Combined Use of } \\
\text { Methylergonovine and Oxytocin during } \\
\text { Caesarean Section in the Prevention of } \\
\text { Post-partum Haemorrhage. }\end{array}$ & $\begin{array}{l}\text { Şentürk, Kağđtçđ, Balđk, } \\
\text { Arslan e Şahin (2016) }\end{array}$ & $\begin{array}{l}\text { Mostrar o benefício do tratamento profilático de } \\
\text { hemorragia pós-parto e a eficácia dos agentes } \\
\text { uterotônicos no uso combinado por infusão de } \\
\text { metilergometrina (metilergonovina) e ocitocina na } \\
\text { prevenção de hemorragia durante e após a cesariana, } \\
\text { em comparação com um grupo de controle que recebeu } \\
\text { infusão de oxitocina apenas. }\end{array}$ & $\begin{array}{l}\text { Um total de } 1.210 \text { pacientes submetidos à cesariana foram incluídos no } \\
\text { estudo. Os critérios de inclusão foram os seguintes: idade entre } 18 \text { anos a } \\
40 \text { anos; gestação } \geq 34 \text { semanas, gestante } 1-3 \text {; e todos com indicações para } \\
\text { cesariana. }\end{array}$ \\
\hline $\begin{array}{c}\text { PUBMED } \\
09 \\
\end{array}$ & $\begin{array}{l}\text { The effect of uterine artery ligation in patients } \\
\text { with central placenta pevia: a randomized }\end{array}$ & & $\begin{array}{l}\text { Avaliar o efeito da ligadura da artéria uterina antes da } \\
\text { incisão uterina em pacientes com placenta prévia }\end{array}$ & agnóstico de placenta pr \\
\hline
\end{tabular}


Research, Society and Development, v. 10, n. 16, e185101622836, 2021

(CC BY 4.0) | ISSN 2525-3409 | DOI: http://dx.doi.org/10.33448/rsd-v10i16.22836

\begin{tabular}{|c|c|c|c|c|}
\hline & controlled trial. / & & central na perda de sangue durante a cesariana. & $\begin{array}{l}\text { recrutados de frequentadores da clínica pré-natal no Minia Maternity } \\
\text { University Hospital no período entre janeiro de } 2014 \text { e dezembro de } 2016 .\end{array}$ \\
\hline $\begin{array}{l}\text { PUBMED } \\
10\end{array}$ & $\begin{array}{l}\text { Uterotonic agents for preventing postpartum } \\
\text { haemorrhage: a network meta-analysis. / }\end{array}$ & Gallos et al. (2018) & $\begin{array}{l}\text { Identificar o (s) agente (s) uterotônico (s) mais eficaz } \\
\text { (is) para prevenir a HPP com o mínimo de efeitos } \\
\text { colaterais e gerar uma classificação de acordo com sua } \\
\text { eficácia e perfil de efeitos colaterais. }\end{array}$ & $\begin{array}{l}\text { Análise de } 196 \text { estudos envolvendo } 135.559 \text { mulheres. Comparamos sete } \\
\text { agentes uterotônicos uns contra os outros e contra mulheres que não } \\
\text { receberam nenhum uterotônico. Os estudos foram realizados em } 53 \text { países. }\end{array}$ \\
\hline $\begin{array}{l}\text { PUBMED } \\
11\end{array}$ & $\begin{array}{l}\text { Effect of early tranexamic acid administration } \\
\text { on mortality, hysterectomy, and other } \\
\text { morbidities in women with post-partum } \\
\text { haemorrhage (WOMAN): na international, } \\
\text { randomised, double-blind, placebo-controlled } \\
\text { trial. / }\end{array}$ & Woman et al. (2017) & $\begin{array}{l}\text { Avaliar os efeitos da administração precoce de ácido } \\
\text { tranexâmico na morte, histerectomia e outros } \\
\text { resultados relevantes em mulheres com hemorragia } \\
\text { pós-parto. }\end{array}$ & $\begin{array}{l}\text { Foram recrutadas mulheres com } 16 \text { anos ou mais com diagnóstico clínico } \\
\text { de HPP após parto vaginal ou cesariana de } 193 \text { hospitais em } 21 \text { países. } \\
\text { Entre março de } 2010 \text { e abril de } 2016,20060 \text { mulheres foram inscritas e } \\
\text { designadas aleatoriamente para receber ácido tranexâmico }(n=10051) \text { ou } \\
\text { placebo }(\mathrm{n}=10009) \text {, das quais } 10036 \text { e } 9985 \text {, respectivamente, foram } \\
\text { incluídas na análise. }\end{array}$ \\
\hline CINAHL 12 & $\begin{array}{l}\text { The effectiveness and safety of introducing } \\
\text { condom-catheter uterine balloon tamponade } \\
\text { for postpartum haemorrhage at secondary } \\
\text { level hospitals in Uganda, Egypt and Senegal: } \\
\text { a stepped wedge, cluster-randomised trial. }\end{array}$ & Anger et al. (2019) & $\begin{array}{l}\text { Avaliar a eficácia da introdução de preservativo-cateter } \\
\text { tamponamento uterino com balão (UBT) para o } \\
\text { tratamento da hemorragia pós-parto (HPP) em } \\
\text { ambientes de baixa e média renda. }\end{array}$ & $\begin{array}{l}\text { Ensaio randomizado por agrupamento em dezoito hospitais de nível } \\
\text { secundário em Uganda, Egito e Senegal, aplicado em mulheres com parto } \\
\text { vaginal de outubro de } 2016 \text { a março de } 2018 \text {. }\end{array}$ \\
\hline CINAHL 13 & $\begin{array}{l}\text { Carbetocin versus rectal misoprostol for } \\
\text { management of third stage of labor among } \\
\text { women with low risk of postpartum } \\
\text { hemorrhage./ }\end{array}$ & Maged et al. (2019) & $\begin{array}{l}\text { Comparar a eficácia e segurança de carbetocina e } \\
\text { misoprostol para prevenção de hemorragia pós-parto } \\
\text { (HPP) entre mulheres de baixo risco. }\end{array}$ & $\begin{array}{l}\text { Ensaio controlado randomizado entre } 150 \text { mulheres grávidas com baixo } \\
\text { risco de HPP admitidas para parto vaginal no Hospital Kasr Al Ainy, } \\
\text { Cairo, Egito, entre julho de } 2018 \text { e maio de } 2019 \text {. }\end{array}$ \\
\hline CINAHL 14 & $\begin{array}{l}\text { Intravenous carbetocin versus intravenous } \\
\text { oxytocin for preventing atonic postpartum } \\
\text { hemorrhage after normal vaginal delivery in } \\
\text { high-risk singleton pregnancies: a triple-blind } \\
\text { randomized controlled trial. }\end{array}$ & $\begin{array}{l}\text { Amornpetchakul et al. } \\
\text { (2018) }\end{array}$ & $\begin{array}{l}\text { Comparar a eficácia da carbetocina intravenosa com a } \\
\text { da ocitocina intravenosa na prevenção da hemorragia } \\
\text { pós-parto atônica (HPP) após parto vaginal em } \\
\text { gestações únicas de alto risco. }\end{array}$ & $\begin{array}{l}\text { Gestantes que deram à luz no Hospital Siriraj entre agosto de } 2016 \text { e } \\
\text { janeiro de } 2017 \text { e que tinham } 20 \text { anos ou mais, idade gestacional de pelo } \\
\text { menos } 34 \text { semanas e parto normal, com pelo menos um fator de risco para } \\
\text { hemorragia pós-parto atônica. }\end{array}$ \\
\hline
\end{tabular}

Fonte: Artigos pesquisados (2021). 


\subsection{Caracterização dos estudos analisados}

Os quatorze estudos fizeram caracterização clínica das intervenções, conforme mostra o quadro 4. Todos os estudos descreveram o tipo de parto onde ocorreu a intervenção no manejo da hemorragia pós-parto, sendo prevalente o parto vaginal (Rangel et al., 2019; Begley et al., 2019; Salati et al., 2019; Asmat et al., 2017; Quibel et al., 2016; Suarez et al., 2020; Escobar et al., 2020; Şentürk et al., 2016; Sanad et al., 2018; Gallos et al., 2018; Woman et al., 2017; Anger et al., 2019; Maged et al., 2019; Amornpetchakul et al., 2018).

Nesse contexto, os seguintes estudos tiveram como critérios de análise o estágio do trabalho de parto para a realização da intervenção, como resultado da análise, o terceiro estágio do trabalho de parto foi o principal período na realização das intervenções (Rangel et al., 2019; Begley et al., 2019; Salati et al., 2019; Asmat et al., 2017; Quibel et al., 2016; Gallos et al., 2018; Maged et al., 2019; Amornpetchakul et al., 2018).

Quanto a perda sanguínea das puérperas na hemorragia pós-parto, os seguintes estudos mostraram que o volume variou de $\geq 500$ a $\geq 2000 \mathrm{~mL}$ (Begley et al., 2019; Salati et al., 2019; Asmat et al., 2017; Quibel et al., 2016; Suarez et al., 2020; Escobar et al., 2020; Şentürk et al., 2016; Sanad et al., 2018; Gallos et al., 2018; Woman et al., 2017; Maged et al., 2019; Amornpetchakul et al., 2018).

Em relação aos fatores de risco e as causas associadas ao desfecho das hemorragias, nos estudos foram reportados: gestantes multíparas, grandes multíparas, placenta prévia, placenta acreta, placenta retida, descolamento prematuro de placenta, atonia uterina, coagulopatia, produtos retidos da concepção, distúrbios hipertensivos e nuliparidade (Asmat et al., 2017; Suarez et al., 2020; Escobar et al., 2020; Sanad et al., 2018; Amornpetchakul et al., 2018).

Um número relativamente pequeno de estudos realizou caracterização sociodemográfica, nos estudos que avaliaram, a faixa etária variou de 18 a 44 anos (Asmat et al., 2017; Şentürk et al., 2016; Sanad et al., 2018; Woman et al., 2017; Anger et al., 2019; Maged et al., 2019; Amornpetchakul et al., 2018).

A respeito das intervenções utilizadas na prevenção da hemorragia pós-parto, nos estudos foram analisadas: ocitocina, misoprostol, ergometrina, carbetocina, metilergonovina, o manejo ativo no terceiro período do trabalho de parto e ligadura da artéria uterina (Rangel et al., 2019; Salati et al., 2019; Begley et al., 2019; Asmat et al., 2017; Quibel et al., 2016; Şentürk et al., 2016; Sanad et al., 2018; Gallos et al., 2018; Maged et al., 2019; Amornpetchakul et al., 2018).

Para o controle da HPP, os estudos analisaram as seguintes intervenções: ocitocina, misoprostol, ácido tranexâmico, tamponamento com balão uterino (BTU) e traje anti-choque não pneumático (NASG) (Rangel et al., 2019; Suarez et al., 2020; Escobar et al., 2020; Woman et al., 2017; Anger et al., 2019).

Acerca da efetividade das intervenções e grau de recomendação, após a avaliação dos quatorze estudos, somente onze obtiveram grau de recomendação A, conforme mostra o quadro 5. As intervenções analisadas neste estudo como efetivas para prevenção e controle estão apresentadas na figura 01 (Rangel et al., 2019; Salati et al., 2019; Asmat et al., 2017; Suarez et al., 2020; Escobar et al., 2020; Şentürk et al., 2016; Sanad et al., 2018; Gallos et al., 2018; Woman et al., 2017; Maged et al., 2019; Amornpetchakul et al., 2018).

De acordo com os 11 estudos avaliados, em relação a efetividade e grau de recomendação A, a ocitocina, o misoprostol e a carbetocina apareceram como as intervenções medicamentosas mais recomendadas e cujos resultados favoráveis foram apresentados em maior número de publicações.

Entre os estudos, dos que analisaram o manejo medicamentoso, três (3/27\%) recomendam o misoprostol e a ocitocina como efetivos na prevenção e controle da hemorragia pós-parto no manejo ativo no terceiro período do trabalho de parto (Rangel et al., 2019; Salati et al., 2019; Asmat et al., 2017); um (1/9\%) recomendou a combinação de misoprostol com ocitocina, ergonomia e carbetocina como intervenções medicamentosas efetivas na prevenção (Gallos et al., 2018); um (1/9\%) recomendou metilergonovina combinada com ocitocina como intervenção efetiva na prevenção (Şentürk et al., 2016); dois 


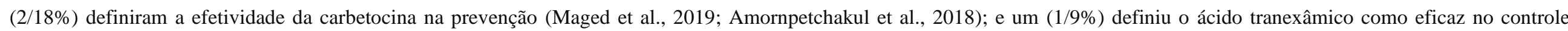
(Woman et al., 2017).

Dos estudos que analisaram o manejo não cirúrgico e cirúrgico, um (1/9\%) definiu o traje anti-choque não pneumático-NASG como efetivo no controle da HPP (Escobar et al.,

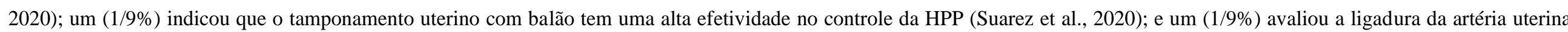
como um método eficaz na prevenção de hemorragia intraoperatória (Sanad et al., 2018).

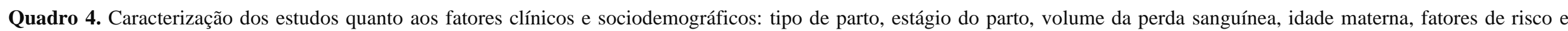
causas. Caxias, MA, Brasil, 2021.

\begin{tabular}{|c|c|c|c|c|c|}
\hline Autor/ano & Tipo de parto & Estágio do parto & Perda sanguínea & Idade materna & Fatores de risco e causas \\
\hline Rangel et al. (2019) & Vaginal & $3^{\circ}$ Estágio & Não Descrito & Não Descrito & Não Descrito \\
\hline Salati et al. (2019) & Vaginal & $3^{\circ}$ Estágio & $\geq 500 \mathrm{a} \geq 1000 \mathrm{~mL}$ & Não Descrito & Não Descrito \\
\hline Begley et al. (2019) & Vaginal & $3^{\circ}$ Estágio & $\geq 500 \mathrm{~mL}$ & Não Descrito & Não Descrito \\
\hline Asmat et al. (2017) & Vaginal & $3^{\circ}$ Estágio & $\geq 500 \mathrm{a}<1000 \mathrm{~mL}$ & $18-42$ anos & Multíparas e Grandes Multíparas \\
\hline Quibel et al. (2016) & Vaginal & $3^{\circ}$ Estágio & $\geq 500 \mathrm{a} \geq 1000 \mathrm{~mL}$ & Não Descrito & Não Descrito \\
\hline Suarez et al. (2020) & Vaginal e Cesáreo & Não Descrito & $\geq 1000 \mathrm{~mL}$ & Não Descrito & $\begin{array}{l}\text { Atonia uterina, placenta prévia, placenta acreta, coagulopatia, } \\
\text { produtos retidos da concepção. }\end{array}$ \\
\hline Escobar et al. (2020) & Vaginal & Não Descrito & $\geq 500 \mathrm{a} \geq 2000 \mathrm{~mL}$ & Não Descrito & $\begin{array}{l}\text { Placenta retida, descolamento prematuro de placenta, atonia } \\
\text { uterina e distúrbios hipertensivos }\end{array}$ \\
\hline Şentürk et al. (2016) & Cesáreo & Não Descrito & $\geq 500 \mathrm{~mL}$ & $18-44$ anos & Não Descrito \\
\hline Sanad et al. (2018) & Cesáreo & Não Descrito & $\geq 500 \mathrm{~mL}$ & $28-38$ anos & Placenta prévia central \\
\hline Gallos et al. (2018) & Vaginal e Cesáreo & $3^{\circ}$ Estágio & $\geq 500 \mathrm{a} \geq 1000 \mathrm{~mL}$ & Não Descrito & Não Descrito \\
\hline Woman et al. (2017) & Vaginal e Cesáreo & Não Descrito & $\geq 500 \mathrm{a} \geq 1000 \mathrm{~mL}$ & $\geq 16$ anos & Não Descrito \\
\hline Anger et al. (2019) & Vaginal & Não Descrito & Não Descrito & $25-35$ anos & Não Descrito \\
\hline Maged et al. (2019) & Vaginal & $3^{\circ}$ Estágio & $\geq 500 \mathrm{~mL}$ & $21-30$ anos & Não Descrito \\
\hline Amornpetchakul et al. (2018) & Vaginal & $3^{\circ}$ Estágio & $\geq 500 \mathrm{~mL}$ & $\geq 20$ anos & Nuliparidade \\
\hline
\end{tabular}

Fonte: Artigos pesquisados (2021). 
Quadro 5. Avaliação das principais intervenções utilizadas no manejo das HPPs, efetividade das intervenções, grau de recomendação e indicação. Caxias, MA, Brasil, 2021.

\begin{tabular}{|c|c|c|c|c|}
\hline Autores/ano & Intervenções avaliadas & Intervenção de maior efetividade & $\begin{array}{c}\text { Grau de } \\
\text { recomendação }\end{array}$ & Indicação \\
\hline Rangel et al. (2019) & $\begin{array}{l}\text { Manejo farmacológico: incluindo estudos sobre ocitocina, misoprostol, } \\
\text { ergometrina, sintometrina, carbetocina, PGF2 } \alpha \text { e ZB11; Manejo ativo no } \\
\text { terceiro período de parto; Tração controlada do cordão; Ocitocina associada } \\
\text { à massagem uterina. }\end{array}$ & $\begin{array}{l}\text { Misoprostol e Ocitocina apresentaram eficácia para a prevenção e o controle da } \\
\text { HPP. O manejo ativo no terceiro período do trabalho de parto com tração } \\
\text { controlada do cordão teve efetividade para a prevenção da HPP. }\end{array}$ & $\mathrm{A}$ & $\begin{array}{l}\text { PREVENÇÃO E } \\
\text { CONTROLE }\end{array}$ \\
\hline Salati et al. (2019) & $\begin{array}{l}\text { A ocitocina profilática em qualquer dose no terceiro estágio do trabalho de } \\
\text { parto comparado com nenhum uterotônico ou placebo. }\end{array}$ & $\begin{array}{l}\text { A ocitocina profilática reduz o risco de perda de sangue e diminui a necessidade } \\
\text { de uterotônicos adicionais. }\end{array}$ & A & PREVENÇÃO \\
\hline Begley et al. (2019) & Manejo ativo versus expectante do terceiro estágio do trabalho de parto. & $\begin{array}{l}\text { O manejo ativo reduziu o risco de HPP primária grave maior que } 1000 \mathrm{~mL} \text { no } \\
\text { momento do nascimento, porém não há certeza desse achado devido às } \\
\text { evidências de qualidade muito baixa. }\end{array}$ & B & PREVENÇÃO \\
\hline Asmat et al. (2017) & Misoprostol $800 \mu \mathrm{gm}$ retal comparado a ocitocina intramuscular 10UI. & $\begin{array}{l}\text { A administração retal de misoprostol } 800 \mu \mathrm{g} \text { foi tão eficaz quanto a ocitocina } \\
10 \mathrm{UI} \text { intramuscular. Ambos foram igualmente eficazes para reduzir a HPP e a } \\
\text { necessidade subsequente de intervenções cirúrgicas. }\end{array}$ & A & PREVENÇÃO \\
\hline Quibel et al. (2016) & $\begin{array}{l}\text { Misoprostol } 400 \mu \mathrm{g} \text { administrado simultaneamente com a ocitocina } \\
\text { intravenosa comparado com placebo por via oral imediatamente no terceiro } \\
\text { estágio do parto. }\end{array}$ & $\begin{array}{l}\text { O misoprostol administrado com ocitocina profilática não reduziu a taxa de } \\
\text { risco de hemorragia pós-parto e aumentou a taxa de eventos adversos. }\end{array}$ & $\mathrm{C}$ & PREVENÇÃO \\
\hline Suarez et al. (2020) & $\begin{array}{l}\text { Compararam o tamponamento uterino com balão versus nenhum } \\
\text { tamponamento uterino com balão na hemorragia pós-parto. }\end{array}$ & $\begin{array}{l}\text { O tamponamento uterino com balão tem uma alta taxa de sucesso no tratamento } \\
\text { de hemorragia pós-parto grave que não responde a uterotônicos e terapias } \\
\text { iniciais, com uma baixa taxa de complicaçóes. }\end{array}$ & A & CONTROLE \\
\hline Escobar et al. (2020) & $\begin{array}{l}\text { Compara os resultados do tratamento em um grupo com um traje anti- } \\
\text { choque não pneumático (NASG) como parte de um pacote de intervenção, } \\
\text { com um grupo de pacientes que não receberam NASG. }\end{array}$ & $\begin{array}{l}\text { NASG associado ao uso de agentes uterotônicos e outras estratégias é uma } \\
\text { ferramenta segura que ajuda a reduzir a morbimortalidade em pacientes graves } \\
\text { com HPP. }\end{array}$ & A & CONTROLE \\
\hline Şentürk et al. (2016) & $\begin{array}{l}\text { Uso combinado de infusão de metilergometrina e ocitocina, em comparação } \\
\text { com um grupo de controle que recebeu infusão apenas de oxitocina. }\end{array}$ & $\begin{array}{l}\text { A metilergometrina combinada com ocitocina foram mais bem-sucedidos do } \\
\text { que os tratamentos com ocitocina apenas, sem qualquer evidência de efeitos } \\
\text { colaterais adversos. }\end{array}$ & A & PREVENÇÃO \\
\hline Sanad et al. (2018) & $\begin{array}{l}\text { A ligadura da artéria uterina antes da incisão uterina para minimizar a perda } \\
\text { de sangue durante a cesariana em pacientes com placenta prévia central } \\
\text { comparada com o grupo controle. }\end{array}$ & $\begin{array}{l}\text { A ligadura da artéria uterina é eficaz para reduzir a perda sanguínea } \\
\text { intraoperatória em pacientes com placenta prévia central submetidas a cesariana } \\
\text { e encurta o tempo operatório. }\end{array}$ & A & PREVENÇÃO \\
\hline Gallos et al. (2018) & $\begin{array}{l}\text { Agentes uterotônicos: oxitocina, misoprostol, ergometrina, carbetocina, } \\
\text { prostaglandinas injetáveis e combinações dessas drogas na prevenção da } \\
\text { HPP, comparados com placebo ou nenhum tratamento. }\end{array}$ & $\begin{array}{l}\text { Os três agentes uterotônicos mais eficazes para prevenção de HPP foram: } \\
\text { ergometrina, combinação de misoprostol mais ocitocina e a carbetocina. }\end{array}$ & A & PREVENÇÃO \\
\hline Woman et al. (2017) & $\begin{array}{l}1 \mathrm{~g} \text { de ácido tranexâmico intravenoso comparado com placebo por injeção } \\
\text { intravenosa lenta. }\end{array}$ & $\begin{array}{l}\text { O ácido tranexâmico reduz a morte por sangramento em mulheres com HPP } \\
\text { sem efeitos adversos. O ácido tranexâmico deve ser administrado o mais rápido } \\
\text { possível após o início do sangramento. }\end{array}$ & A & CONTROLE \\
\hline Anger et al. (2019) & $\begin{array}{l}\text { Tamponamento com balão uterino de preservativo-cateter (BTU) para o } \\
\text { tratamento de hemorragia pós-parto (HPP). }\end{array}$ & $\begin{array}{l}\text { A introdução do BTU não reduz a incidência combinada de cirurgia ou morte } \\
\text { relacionada à HPP. }\end{array}$ & B & CONTROLE \\
\hline Maged et al. (2019) & $\begin{array}{l}\text { Carbetocina uma ampola de }(100 \mu \mathrm{g} / \mathrm{mL}) \text { por via intravenosa, e o } \\
\text { misoprostol, dois comprimidos retais de }(800 \mu \mathrm{g}) \text { para manejo ativo do } \\
\text { terceiro estágio. }\end{array}$ & $\begin{array}{l}\text { A carbetocina foi mais efetiva para o manejo ativo da terceira fase do trabalho } \\
\text { de parto entre mulheres de baixo risco, reduziu a perda de sangue e o uso de } \\
\text { drogas uterotônicas adicionais. }\end{array}$ & A & PREVENÇÃO \\
\hline $\begin{array}{l}\text { Amornpetchakul et } \\
\text { al. (2018) }\end{array}$ & $\begin{array}{l}\text { Carbetocina } 100 \text { mcg comparada com a ocitocina 5UI por via intravenosa, } \\
\text { na prevenção da hemorragia pós-parto atônica (HPP) após parto vaginal. }\end{array}$ & $\begin{array}{l}\text { A carbetocina intravenosa é mais eficaz do que a ocitocina intravenosa na } \\
\text { prevenção da HPP atônica em gestações únicas com pelo menos um fator de } \\
\text { risco para HPP. }\end{array}$ & $\bar{A}$ & PREVENÇÃO \\
\hline
\end{tabular}

Fonte: Artigos pesquisados (2021) 
Figura 1. Fluxograma relacionado à identificação das intervenções efetivas na prevenção e controle das hemorragias pósparto. Caxias, MA, Brasil, 2021.

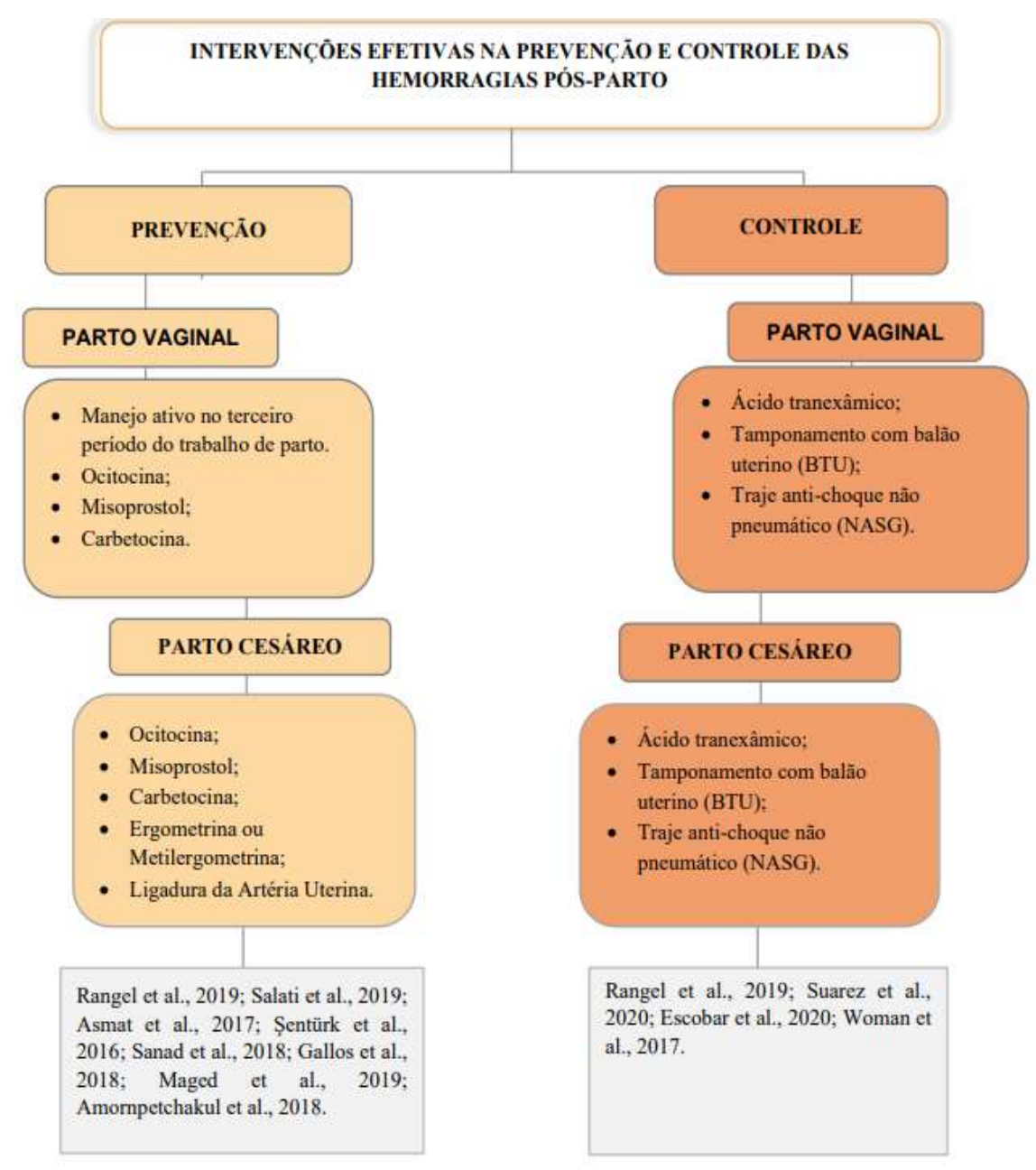

Fonte: Artigos pesquisados (2021).

\section{Discussão}

\subsection{Caracterização dos estudos das intervenções quanto aos fatores clínicos e sociodemográficos}

Foram encontrados diversos tipos de intervenções utilizadas no manejo das hemorragias pós-parto, nos estudos dessa pesquisa predominaram às análises do manejo farmacológico, também havendo análises de procedimentos de manejo cirúrgico e não cirúrgico. As análises de intervenção foram realizadas em hospitais, com prevalência em mulheres que tiveram parto vaginal, quanto ao momento da intervenção, o terceiro estágio do trabalho de parto foi relatado como o período mais crítico e oportuno. As mulheres recrutadas para os estudos incluídos tiveram média de idade entre 18 a 44 anos, a perda sanguínea variou de $\geq 500$ a $\geq 2000 \mathrm{~mL}$ corroborando com os estudos de Lumbreras-Marquez et al. (2020), onde as parturientes apresentavam as mesmas características.

Embora um dos objetivos desta análise considere caracterizar os fatores de risco relacionados ao desfecho das hemorragias pós-parto, a maioria dos estudos caracterizaram as mulheres independentemente dos fatores de risco de sangramento, alguns estudos adotaram como critérios de exclusão mulheres com histórico de HPP em partos anteriores e mulheres com alto risco de sangramento.

Nos estudos de Asmat et al. (2017) e Suarez et al. (2020), que incluíram pacientes com fatores de risco de hemorragia pós-parto em suas análises, reportaram como mais prevalentes: gestantes multíparas, grandes multíparas, placenta prévia, 
placenta acreta, coagulopatia e produtos retidos da concepção. Escobar et al. (2020) em seu estudo incluíram pacientes que apresentaram placenta retida, descolamento prematuro de placenta, atonia uterina e distúrbios hipertensivos.

Corroborando com os resultados deste estudo, Bienstock et al. (2021), analisaram que as principais causas de hemorragia pós-parto são atonia uterina, lacerações, retenção de placenta ou coágulos e deficiência de fator de coagulação. Em contrapartida, os estudos de Feduniw et al. (2020) e Bi et al. (2021), afirmam que uma grande quantidade das HPPs é causada pela ocorrência de atonia uterina secundária a um aumento das taxas de cesarianas, e que lamentavelmente, a hemorragia ocorre em dois terços das mulheres sem quaisquer fatores de risco. É comumente sabido que é melhor prevenir do que tratar possíveis complicações.

Salati et al. (2019), reforça a ideia e afirma que estudos futuros de revisão sistemática devem ter como objetivo incluir fatores de risco e desfechos importantes, como mortalidade materna, choque, transferência para um nível superior de atendimento, efeitos colaterais graves e outros desfechos centrados na paciente como satisfação com a terapia e resultados neonatais, analisando todos os dados disponíveis.

\subsection{Identificação as principais intervenções utilizadas na prevenção e no controle da hemorragia pós-parto}

Os estudos analisaram as seguintes intervenções na prevenção da hemorragia pós-parto: ocitocina, misoprostol, carbetocina, ergometrina, metilergometrina, o manejo ativo no terceiro período do trabalho de parto e a ligadura da artéria uterina. Assim como os resultados apresentados no estudo de Liabsuetrakul, Choobun, Islam e Peeyananjarassri (2018), existem muitas variações possíveis a respeito do pacote de intervenções para HPP. Diferentes drogas uterotônicas podem ser utilizadas, por exemplo, a ocitocina, misoprostol, ergometrina.

Nessa perspectiva, os estudos de Gallos et al., 2018, ressaltam que no parto vaginal, a administração de um agente uterotônico costuma ser um componente do manejo ativo do terceiro estágio do trabalho de parto, junto com o clampeamento precoce do cordão e a tração controlada do cordão. Corroborando com a afirmativa acima, o estudo de Salati et al. (2019) complementa, que a ocitocina profilática pode ser considerada como um componente do manejo ativo do terceiro estágio do parto.

Na fisiologia do parto, a hemorragia pós-parto é evitada por contraç̃os miometriais que começam com a separação da placenta e participação de hormônios. De acordo com Şentürk et al. (2016), a promoção de contrações miometriais se tornou o principal objetivo dos tratamentos. Muitas vezes a HPP ocorre na ausência de fatores de risco clínicos ou históricos identificáveis. Portanto, a prevenção eficaz é defendida para todas as mulheres durante o parto.

No estudo de Gaucher et al. (2019), complementam que melhorar a prevenção deve reduzir as taxas gerais (menores ou maiores) de HPP, enquanto uma gestão melhorada deve reduzir complicações importantes, especialmente mortalidade e morbidades graves.

No que se refere as intervenções para o controle da hemorragia pós-parto, os estudos analisaram: ocitocina, misoprostol, ácido tranexâmico, tamponamento com balão uterino (BTU) e traje anti-choque não pneumático (NASG). Assim como os resultados apresentados no estudo de Kellie, Wandabwa, Mousa e Weeks (2020) ao analisarem às intervenções mecânicas e cirúrgicas para o tratamento de hemorragia pós-parto primária.

\subsection{Avaliação do grau de recomendação e a efetividade das intervenções na prevenção e controle das HPPS}

As intervenções com grau de recomendação A, mais efetivas de acordo com os estudos incluídos nesta revisão foram medicamentosas, a ocitocina, o misoprostol e a carbetocina, apareceram como as intervenções mais recomendadas e cujos resultados favoráveis foram apresentados em maior número de publicações. Corroborando com as evidências de outros 
estudos, como os realizados por Adnan, Conlan-Trant et al. (2018), Aziz et al. (2018), Kuzume et al. (2017), no entanto as dosagens, regimes e vias de administração dos agentes uterotônicos mais eficazes variaram.

Nos estudos que investigaram esses agentes, a ocitocina foi administrada por via intramuscular (IM) ou intravenosa (IV) em doses variando de 5 unidades internacionais (IU) a $10 \mathrm{IU}$, com alguns estudos sem esclarecimento se a via IV foi administrada por bolus ou infusão em soro, conforme apresentaram os estudos de Salati et al. (2019) e Asmat et al. (2017).

Relacionado com esses achados, os estudos de Oladapo et al. (2018) e Carvalho et al. (2020), indicam que não há diferença clara entre os benefícios e riscos comparativos da ocitocina intramuscular e intravenosa quando administrada para prevenir a perda excessiva de sangue após o parto vaginal. A ocitocina reduziu significativamente a perda de sangue e a incidência de HPP durante partos vaginais, sem um aumento nos efeitos adversos.

Em contrapartida, os resultados divergem nos estudos realizados por Adnan et al. (2018) e Wu et al. (2020), que constataram que a ocitocina intravenosa é uma opção boa e segura no manejo do terceiro estágio do parto, que resultou em HPP grave menos frequente, transfusão de sangue e admissão em uma unidade de alta dependência do que a ocitocina intramuscular, e sem efeitos colaterais excessivos.

Nos estudos que avaliaram o misoprostol combinado com ocitocina, as doses e vias variaram muito, com alguns estudos administrando uma infusão intravenosa de 20 UI de ocitocina e $400 \mathrm{mcg}$ de misoprostol por via sublingual, ou 200 mcg de misoprostol por via sublingual, enquanto outros administraram uma infusão intravenosa de 10 UI de ocitocina e 400 mcg de misoprostol por via retal.

Asmat et al. (2017), em seu estudo avaliaram a eficácia do misoprostol retal em relação à ocitocina na hemorragia pós-parto primária em um total de 1.678, e evidenciou que ambos foram igualmente efetivos na prevenção de hemorragias. Os resultados do estudo de Quibel et al., (2016), divergem ao concluírem que o misoprostol administrado com ocitocina profilática não reduziu a taxa de risco de hemorragia pós-parto e aumentou a taxa de eventos adversos.

Quanto ao uso de carbetocina, foi administrada como um único bolus intravenoso de $100 \mathrm{mcg}$ ou por via intramuscular. Os achados deste estudo, corroboram com o que relata Aziz et al. (2018), que mostrou que a carbetocina é eficaz para diminuir a HPP, a perda de sangue, a duração do terceiro estágio do trabalho de parto e a necessidade de uterotônicos adicionais. Gallos et al. (2018) em sua análise, concluíram que todos os uterotônicos foram eficazes para prevenir a HPP, mas que a carbetocina pode ser mais eficaz que a oxitocina sem aumentar os efeitos colaterais, para prevenir HPP de $500 \mathrm{~mL}$.

Outras intervenções medicamentosas avaliadas com grau de recomendação A, foram ergometrina ou metilergometrina e ácido tranexâmico. No estudo de Şentürk et al. (2016), os tratamentos profiláticos de metilergometrina combinados de ocitocina foram mais bem-sucedidos do que os tratamentos com apenas ocitocina e significativamente sem qualquer evidência de efeitos colaterais adversos. A administração de metilergometrina como um tratamento uterotônico combinado para profilaxia de hemorragia pós-parto pode ser tão segura e eficiente quanto os outros tratamentos uterotônicos. Esses dados apresentam similaridade nos achados de Rangel et al., 2019.

O ácido tranexâmico é um medicamento que reduz o sangramento ao inibir a quebra dos coágulos sanguíneos de fibrina. Quando usado como tratamento para hemorragia pós-parto, deve ser administrado o mais rápido possível após o início do sangramento. A efetividade do ácido tranexâmico na redução do risco de morte por hemorragia pós-parto foi demonstrado no ensaio de Woman et al. (2017). Desse modo, Pacheco et al. (2017) complementa que a dose usada na maioria dos estudos é de $1 \mathrm{~g}$ administrado IV.

Os achados relacionados às intervenções não-cirurgicas e cirúrgicas com grau de recomendação A, destacaram o traje anti-choque não pneumático (NASG), o tamponamento com balão uterino (BTU) e a ligadura da artéria uterina. 
Os achados Escobar et al. (2020), mostram que o traje anti-choque não pneumático é um dispositivo indicado para pacientes com HPP acompanhada de algum grau de choque. Sua utilidade tem sido demonstrada em centros sem hemoderivados e pessoal cirúrgico especializado, como estratégia de transferência segura para unidades de segundo e terceiro nível, para estabilizar a paciente que será operada ou como medida complementar pós-operatória em casos de hemorragia obstétrica maciça. Essas evidências corroboram com os resultados dos estudos de Meade-Treviño, Fernández-Lara, LizaolaDíaz de Leon, Mendoza-Mares e Grimaldo-Valenzuela (2021) e Mbaruku et al. (2018).

O estudo de Suarez et al. (2020), demonstrou alta taxa de sucesso do BTU na contenção do sangramento entre mulheres com HPP grave que não respondem a uterotônicos e terapias iniciais. Além disso, as evidências sugerem que o uso de BTU está associado a uma redução significativa na taxa de procedimentos invasivos relacionados a HPP, como ligadura de artéria, suturas de compressão uterina, histerectomia e embolização arterial.

Resultados semelhantes foram encontrados por Darwish et al. (2017). O que contrapõe os resultados do estudo de Anger et al. (2019), que concluiu que a introdução de BTU com preservativo-cateter não melhorou os resultados maternos e foi associada a um aumento na incidência combinada de cirurgia relacionada à HPP e morte materna.

Lin, Lin e Zhang (2019), ao realizarem um estudo avaliando a ligadura bilateral da artéria uterina antes do parto na prevenção de HPP, em mulheres com placenta acreta, concluíram que pode reduzir efetivamente a quantidade de perda sanguínea intra-operatória, a incidência de HPP e o risco de complicações, como histerectomia. Corroborando com os resultados de Sanad et al., 2018 incluído neste estudo.

Durante a busca nas bases de dados, não foram encontrados artigos de estudos que demonstrassem certeza da superioridade de um tratamento sobre o outro na profilaxia ou tratamento da hemorragia pós-parto, não sendo possível chegar a um consenso sobre a melhor abordagem, o que representa uma limitação deste estudo.

\section{Considerações Finais}

Este estudo permitiu conhecer as evidências acerca das intervenções mais efetivas utilizadas para a prevenção e o controle da hemorragia pós-parto, as analisadas como efetivas na prevenção foram: ocitocina, misoprostol, carbetocina, ergometrina ou metilergometrina, o manejo ativo no terceiro período do trabalho de parto e a ligadura da artéria uterina. No controle foram: o ácido tranexâmico, tamponamento com balão uterino (BTU) e o traje anti-choque não pneumático (NASG). Considerando a necessidade que as puérperas têm de um suporte tecnológico rápido e resolutivo dessas intervenções e que podem ser utilizadas na pratica clínica.

Atualmente, maior atenção foi dada à hemorragia pós-parto, por ser a segunda maior causa de morte materna no Brasil. A hemorragia pós-parto pode ser prevenida, conforme demonstram as evidências encontradas na literatura discutidas neste estudo, desde que as melhores intervenções sejam incorporadas a prática clínica. As mortes maternas devem ser reduzidas, pois a HPP é um evento adverso evitável através de ações para o parto seguro e por ser um compromisso social assumido pelo governo brasileiro.

Existe uma carência em relação a quantidade de produções cientificas de alto nível de evidência acerca desta temática. As evidências inclusas nesta revisão foram insuficientes para analisar todas as intervenções disponíveis na prevenção e controle da HPP, determinar a efetividade relativa, a via, a dose e a segurança, bem como efeitos adversos e contraindicações das intervenções que compuseram a amostra desse estudo. Outros estudos são necessários para determinar essas variáveis.

Os objetivos realizados no decorrer desse estudo foram alcançados, demonstrados nos quadros e no fluxograma. Importante enfatizar que uma boa assistência de enfermagem no pré-natal, na atenção hospitalar, contribui de forma significativa para um parto seguro, e o sucesso da equipe no cuidado integral as pacientes. Os enfermeiros na prática clínica 
devem pesquisar constantemente, incorporar as evidências científicas como suporte a suas práticas, construir protocolos de enfermagem para oferecer às mulheres os melhores cuidados.

\section{Referências}

Adnan, N., Conlan-Trant, R., Mccormick, C., Boland, F., \& Murphy, D. J. (2018). Intramuscular versus intravenous oxytocin to prevent postpartum haemorrhage at vaginal delivery: randomised controlled trial. Bmj, 362, 3546.

Amornpetchakul, P., Lertbunnaphong, T., Boriboonhiransarn, D., Leetheeragul, J., Sirisomboon, R., \& Jiraprasertwong, R. (2018). Intravenous carbetocin versus intravenous oxytocin for preventing atonic postpartum hemorrhage after normal vaginal delivery in high-risk singleton pregnancies: a triple-blind randomized controlled trial. Archives Of Gynecology And Obstetrics, 298(2), 319-327.

Anger, H., Dabash, R., Durocher, J., Hassanein, N., Ononge, S., Frye, L., \& Darwish, E. (2019). The effectiveness and safety of introducing condom-catheter uterine balloon tamponade for postpartum haemorrhage at secondary level hospitals in Uganda, Egypt and Senegal: a stepped wedge, cluster : randomised trial. Bjog: An International Journal of Obstetrics \& Gynaecology, 12613, 1612-1621.

Asmat, R., Ashraf, T., Asmat, S., Asmat, N., \& Asmat, F. (2017). Effectiveness of Per Rectal Misoprostol Versus Intramuscular Oxytocin for Prevention of Primary Postpartum Haemorrhage. Journal Of The College Of Physicians And Surgeons, 27(1), 13-17.

Aziz, M. A. A. E., Iraqi, A., Abedi, P., \& Jahanfar, S. (2018). The effect of carbetocin compared to misoprostol in management of the third stage of labor and prevention of postpartum hemorrhage: a systematic review. Systematic Reviews, 7(1), 170-177.

Begley, C. M., Devane, D., Murphy, D. J., Gyte, G. M., Mcdonald, S. J., \& Mcguire, W. (2019). Active versus expectant management for women in the third stage of labour. Cochrane Database Of Systematic Reviews, 1-142.

Bi, S., Zhang, L., Chen, J., Huang, M., Huang, L., Zeng, S., \& Wen, Suiwen. (2021). Maternal age at first cesarean delivery related to adverse pregnancy outcomes in a second cesarean delivery: a multicenter, historical, cross-sectional cohort study. Bmc Pregnancy And Childbirth, $21,1-10$.

Bienstock, J. L., Eke, A. C., Hueppchen, N. A. (2021). Postpartum Hemorrhage. New England Journal Of Medicine, 384(17), 1635-1645.

Bork, A.M.T. (2005). Enfermagem baseada em evidencias. Guanabara Koonga.

Carvalho, N., Hoque, M. E., Oliver, V. L., Byrne, A., Kermode, M., Lambert, P., \& Morgan, A. (2020). Cost-effectiveness of inhaled oxytocin for prevention of postpartum haemorrhage: a modelling study applied to two high burden settings. Bmc Medicine, 18(1), 8-18.

Darwish, A. M., Abdallah, M. M., Shaaban, O. M., Ali, M. K., Khalaf, M., \& Sabra, A. M. A. (2017). Bakri balloon versus condom-loaded Foley’s catheter for treatment of atonic postpartum hemorrhage secondary to vaginal delivery: a randomized controlled trial. The Journal Of Maternal-Fetal \& Neonatal Medicine, 31(6), 747-753.

Ercole, F. F., Melo, L. S., \& Alcoforado, C. L. G. C. (2014) Revisão integrativa versus revisão sistemática. Revista Mineira de Enfermagem, $18(1)$, 9-11.

Escobar, M. F., Pérez, P. A. F., Carvajal, J. A., Burgos, J. M., Messa, A., Echavarria, M. P., \& Hurtado, D. F. (2020). Impact of nonpneumatic antishock garment in the management of patients with hypoperfusion due to massive postpartum hemorrhage. The Journal Of Maternal-Fetal \& Neonatal Medicine, 33(18), 3086-3090.

Feduniw, S., Warzecha, D., Szymusik, I., \& Wielgos, M. (2020). Epidemiology, prevention and management of early postpartum hemorrhage — a systematic review. Ginekologia Polska, 91(1), 38-44.

Felipe, A. C. C., Ansaloni, L. V. S., Martins, M. V., Sousa, M. J. D., \& Oliveira, R. A. D. (2020). Fatores assistenciais que influenciam nos altos índices de mortalidade materna por hemorragia puerperal. Revista de Divulgação Científica Sena Aires, 9(3), 551-562.

Finlayson, K., Downe, S., Vogel, J.P., \& Oladapo, O. T. (2019). What matters to women and healthcare providers in relation to interventions for the prevention of postpartum haemorrhage: A qualitative systematic review. PLoS ONE, 2019. 14 (5), e0215919.

Gallos, I. D., Williams, H. M., Price, M. J., Merriel, A., Gee, H., Lissauer, D., \& Coomarasamy, A. (2018). Uterotonic agents for preventing postpartum haemorrhage: a network meta-analysis. Cochrane Database Of Systematic Reviews, 1-362.

Gaucher, L., Occelli, P., Deneux-Tharaux, C., Colin, C., Gaucherand, P., Touzet, S., \& Dupont, C. (2019). Non-clinical interventions to prevent postpartum haemorrhage and improve its management: a systematic review. European Journal Of Obstetrics \& Gynecology And Reproductive Biology, $240,300-309$.

Kellie, F. J., Wandabwa, J. N., Mousa, A. H., Weeks, A. D. (2020). Mechanical and surgical interventions for treating primary postpartum haemorrhage. Cochrane Database Of Systematic Reviews, 1-91.

Kuzume, A., Sugimi, S., Suga, S., Yamashita, H., \& Yasuhi, I. (2017). The Routine Use of Prophylactic Oxytocin in the Third Stage of Labor to Reduce Maternal Blood Loss. Journal Of Pregnancy, 2017, 3274901.

Liabsuetrakul, T., Choobun, T., Islam, M., \& Peeyananjarassri, K. Prophylactic use of ergot alkaloids in the third stage of labour. Cochrane Database Of Systematic Reviews, 1-62.

Lin, J., Lin, F., \& Zhang, Y. (2019). Uterine artery ligation before placental delivery during caesarean in patients with placenta previa accreta. Medicine, 98(36), 16780. 
Lumbreras-Marquez, M. I., Reale, S. C., Carusi, D. A., Robinson, J. N., Scharf, N., Fields, K. G., \& Farber, M. K. (2020). Introduction of a Novel System for Quantitating Blood Loss After Vaginal Delivery. Anesthesia \& Analgesia, 130(4), 857-868.

Maged, A. M., Waly, M., Fahmy, R. M., Dieb, A. S., Essam, A., Salah, N. M., Hussein, E. A., \& Nabil, H. (2019). Carbetocin versus rectal misoprostol for management of third stage of labor among women with low risk of postpartum hemorrhage. International Journal Of Gynecology \& Obstetrics, 148(2), 238242.

Martins, A. C. S., \& Silva, L. S. (2018). Perfil epidemiológico da mortalidade materna. Revista Brasileira de Enfermagem, 71, 677-683.

Mbaruku, G., Therrien, M. S., Tillya, R., Mbuyita, S., Mtema, Z., Kinyonge, I., \& Miller, S. (2018). Implementation project of the non-pneumatic anti-shock garment and m-communication to enhance maternal health care in rural Tanzania. Reproductive Health, $15,177$.

Meade-Treviño, P. F., Fernández-Lara, J. A., Lizaola-Díaz de Leon, H., Mendoza-Mares, R, \& Grimaldo-Valenzuela, P. M. (2021). Vestuário anti-choque não pneumático: uma intervenção valiosa no tratamento de uma paciente com hemorragia obstétrica. Ginecología y Obstetricia de México, 86(3), $200-207$.

Oladapo, O. T., Okusanya, B. O., \& Abalos, E. (2018). Intramuscular versus intravenous prophylactic oxytocin for the third stage of labour. Cochrane Database Of Systematic Reviews, 1-37.

Oliveira, F. B. M., Costa, A. C. A. L., Alves, D. L., De França, J. F., Macedo, M. S., \& Santos, R. D. (2016). Relação entre a sobrecarga de trabalho e erros de administração de medicação na assistência hospitalar. ReonFacema, 2(2):325-334.

Oliveira, R. C., \& Davim, R. M. B. (2019). Prevenção e tratamento da hemorragia pós-parto. Rev Enferm Ufpe, 13, $236-248$.

Pacheco, L. D., Hankins, G. D. V., Saad, A. F., Costantine, M. M., Chiossi, G., Saade, G. R. (2017). Tranexamic Acid for the Management of Obstetric Hemorrhage. Obstetrics \& Gynecology, 130(4), 765-769.

Quibel, T., Ghout, I., Goffinet, F., Salomon, L. J., Fort, J., Javoise, S., . . \& Rozenberg, P. (2016). Active Management of the Third Stage of Labor With a Combination of Oxytocin and Misoprostol to Prevent Postpartum Hemorrhage. Obstetrics \& Gynecology, 128, (4), 805-811.

Rangel, R. C. T., Souza, M. L., Bentes, C. M. L., Souza, A. C. R. H., Leitão, M. N. C., \& Lynn, F. A. (2019). Tecnologias de cuidado para prevenção e controle da hemorragia no terceiro estágio do parto: revisão sistemática. Rev. Latino-Americana de Enfermagem, $27,3165$.

Salati, J. A., Leathersich, S. J., Williams, M. J., Cuthbert, A., \& Tolosa, J. (2019). Prophylactic oxytocin for the third stage of labour to prevent postpartum haemorrhage. Cochrane Database Of Systematic Reviews, 1-95.

Sanad, A S., Mahran, A. E., Aboulfotouh, M. E., Kamel, H. H., Mohammed, H. F., Bahaa, \& Shawki, H. E.-D. (2018). The effect of uterine artery ligation in patients with central placenta pevia: a randomized controlled trial. Bmc Pregnancy And Childbirth, $18,351$.

Şentürk, Ş., Kağđtçđ, M., Balđk, G., Arslan, H., \& Şahin, F. K. (2016). The Effect of the Combined Use of Methylergonovine and Oxytocin during Caesarean Section in the Prevention of Post-partum Haemorrhage. Basic \& Clinical Pharmacology \& Toxicology, 118(5), 338-343.

Suarez, S., Conde-Agudelo, A., Borovac-Pinheiro, A., Suarez-Rebling, D., Eckardt, M., Theron, G., \& Burke, T. F. (2020). Uterine balloon tamponade for the treatment of postpartum hemorrhage: a systematic review and meta-analysis. American Journal Of Obstetrics And Gynecology, $222(4), 293$.

Woman, T. C., Shakur, H., Roberts, I., Fawole, B., Chaudhri, R., El-Sheikh, M., \& Abdulkadir, A. (2017). Effect of early tranexamic acid administration on mortality, hysterectomy, and other morbidities in women with post-partum haemorrhage (WOMAN): an international, randomised, double-blind, placebocontrolled trial. The Lancet, 389(10084), 2105-2116.

World Health Organization. (2018). World Health Organization multicountry survey on maternal and newborn health. Geneva: WHO.

Wu, Y., Wang, H., Wu, Q.-Y., Liang, X.-L., \& Wang, J. (2020). A meta-analysis of the effects of intramuscular and intravenous injection of oxytocin on the third stage of labor. Archives Of Gynecology And Obstetrics, 301(3), 643-653. 